\title{
Infinitely many solutions for a class of hemivariational inequalities involving $\mathrm{p}(\mathrm{x})$-Laplacian
}

\author{
Fariba Fattahi, Mohsen Alimohammady
}

\begin{abstract}
In this paper hemivariational inequality with nonhomogeneous Neumann boundary condition is investigated. The existence of infinitely many small solutions involving a class of $p(x)$-Laplacian equation in a smooth bounded domain is established. Our main tool is based on a version of the symmetric mountain pass lemma due to Kajikiya and the principle of symmetric criticality for a locally Lipschitz functional.
\end{abstract}

\section{Introduction}

We are concerned with the study of the nonlinear elliptic differential inclusion involving the $p(x)$-Laplacian equation

$$
\begin{cases}-\Delta_{p(x)} u+|u|^{p(x)-2} u=\lambda|u|^{q(x)-2} u & \text { in } \Omega \\ -|\nabla u|^{p(x)-2} \frac{\partial u}{\partial \nu} \in-\partial F(x, u) & \text { on } \partial \Omega,\end{cases}
$$

where $\Omega \subset \mathbb{R}^{N}(N \geq 2)$ is a bounded smooth domain, $\frac{\partial u}{\partial \nu}$ is the outer unit

Key Words: $\mathrm{p}(\mathrm{x})$-Laplacian, symmetric mountain pass lemma, hemivariational inequality, generalized Lebesgue-Sobolev spaces.

2010 Mathematics Subject Classification: Primary 49J40, 47J30; Secondary 35B30, $35 \mathrm{~J} 60$.

Received: 07.07.2016

Revised: 10.09.2016

Accepted: 19.09.2016 
normal derivative on $\partial \Omega$.

$p, q: \bar{\Omega} \rightarrow \mathbb{R}$ are continuous functions satisfying

$$
1<p^{-}=\min _{x \in \bar{\Omega}} p(x) \leq p(x) \leq p^{+}=\max _{x \in \bar{\Omega}} p(x)<+\infty
$$

such that $\inf _{x \in \bar{\Omega}}(q(x)-p(x))>0, \lambda$ is a real parameter, $\lambda<\frac{q^{-} \lambda_{*}}{p^{+}} . F: \Omega \times \mathbb{R} \rightarrow$ $\mathbb{R}$ is locally Lipschitz function given by $F(x, \omega)=\int_{0}^{\omega} f(x, t) d t, \omega \in \mathbb{R}$ such that $f: \partial \Omega \times \mathbb{R} \rightarrow \mathbb{R}$ is locally essentially bounded function. $\partial F(x, u)$ denote the generalized Clarke gradient of $F(x, u)$.

$u \in X:=W_{0}^{1, p(\cdot)}(\Omega)$ is said to be a weak solution of problem (1.1), if

$$
\begin{aligned}
& \int_{\Omega}|\nabla u(x)|^{p(x)-2} \nabla u(x) \nabla v(x) d x+\int_{\Omega}|u|^{p(x)-2} u v d x \\
= & \lambda \int_{\Omega}|u(x)|^{q(x)-2} u(x) v(x) d x+\int_{\partial \Omega} g^{*} v(x) d \sigma, \quad \forall v \in X,
\end{aligned}
$$

with $g^{*} \in L^{r^{\prime}(x)}(\Omega)$ (for some $r \in\left(p, p^{*}\right), \frac{1}{r(x)}+\frac{1}{r^{\prime}(x)}=1$ and $g^{*}(x) \in$ $\partial F(x, u(x))$. is

We point out $\lambda_{*}$ is the Rayleigh quotient associated with our problem, that

$$
\lambda_{*}:=\inf _{u \in W_{0}^{1, p(x)}(\Omega) \backslash\{0\}} \frac{\int_{\Omega} \frac{1}{p(x)}|\nabla u|^{p(x)} d x+\int_{\Omega} \frac{1}{p(x)}|u|^{p(x)} d x}{\int_{\Omega} \frac{1}{q(x)}|u|^{q(x)} d x} .
$$

The operator $\Delta_{p(x)} u=\operatorname{div}\left(|\nabla u|^{p(x)-2} \nabla u\right)$ is the so-called $p(x)$-Laplacian which becomes $p$-Laplacian when $p(x) \equiv p$ is a constant. In particular, we refer on the applications to $p(x)$-Laplacian problems in (cf. [21], [2], [7]). However, these papers are considered the application of the main results in order to achieve the existence of infinitely many solutions, but did not give more information on the sequence of solutions. This difficulty was overcome by Kajikiya who, in (cf. [14]) presented the definitive version of the infinitely many points related to the symmetric mountain pass lemma and applied to a sublinear elliptic equation.

In recent years, differential equations and variational problems have been studied in many papers, we refer to some interesting works (cf. [24], [25]).

Indeed, the investigation of existence and multiplicity of solutions for problems with $p(x)$-Laplacian operators via Kajikiya lemma has drawn the attentions of many authors; In (cf. [15]) authors studied the existence of at least one solution and infinitely many solutions of $p(x)$-biharmonic problem by using mountain pass theorem, fountain theorem, symmetric mountain pass lemma due to Kajikiya; In (cf. [16]) authors studied the existence and multiplicity 
of solutions to the fractional Kirchhoff-type problem by using critical point theorems and the truncation technique; In (cf. [17]) authors studied the existence and multiplicity of solutions for the problem Kirchhoff-type problem by variational methods; In (cf. [18]) authors studied the existence of infinitely many solutions in a bounded domain via variational tools.

In the past decades, the existence and uniqueness of solutions to several classes of nonlinear inclusions for variational and hemivariational inequalities were considered. These types of inequalities have established a new subject in nonsmooth analysis, due to these fields are based on the subdifferential in the sense of Clarke of locally Lipschitz functionals. The theory of hemivariational inequalities has given significant consequence both in pure and applied mathematics and it is valuable to comprehend many problems of mechanics and engineering for non-convex, non-smooth functionals.

The applications to non-smooth variational problems have been seen in many papers, in (cf. [4]) authors studied the existence of infinitely many solutions for variational-hemivariational inequalities with nonhomogeneous Neumann boundary conditions; in (cf. [12]) authors studied the existence of infinitely many anti-periodic solutions for a second-order impulsive differential inclusion problem for non-smooth functionals; in (cf. [13]), author studied the existence of infinitely many radial respective non-radial solutions for a class of hemivariational inequalities; in (cf. [23]), authors studied variational-hemivariational inequalities for the existence of a whole sequence of solutions with non-smooth potential and non-zero Neumann boundary condition; in (cf. [5]), authors studied variational-hemivariational inequalities involving the p-Laplace operator and a nonlinear Neumann boundary condition; in (cf. [1]), authors studied variational-hemivariational inequality by using the mountain pass theorem for the existence of at least one solution to a boundary value problem involving the $p(x)$-biharmonic operator.

In the present paper, we extend the existence of infinitely many arbitrarily small solutions converging to zero by new version of the symmetric mountain pass lemma due to Kajikiya (cf. [14]) to a class of locally Lipschitz functionals. Motivated by reasons above, we prove the existence of infinitely many solutions for a hemivariational inequality depending on one parameter for a differential inclusion $p(x)$-Laplacian problem. The main tool used in our paper is applying the non-smooth version of the symmetric mountain pass lemma is the principle of criticality for a locally Lipschitz functional.

We start the paper by giving in section 2 the properties of the generalized Sobolev space. Also we present some definitions and properties for the generalized gradient of the locally Lipschitz functionals. The main part is concerned with the existence of infinitely many solutions for a class of nonlinear hemivariational inequalities on bounded domain by using non-smooth symmetric 
mountain pass lemma for locally Lipschitz functionals.

\section{Preliminaries}

In order to deal with the problem (1.1), we need some theory of variable exponent Sobolev space. We prepare the basic definitions and properties in the framework of the generalized Lebesgue and Sobolev spaces (cf. [9], [10], [11]). For convenience, we only recall some basic facts which will be used later. Let $\Omega \subset \mathbb{R}^{N}$ a bounded domain. Denote

$$
C_{+}(\bar{\Omega})=\{p(x) ; p(x) \in C(\bar{\Omega}), p(x)>1, \text { for all } x \in \bar{\Omega}\} .
$$

For any Lipschitz continuous function $p: \Omega \rightarrow(1, \infty)$ denote by $1<p^{-}=$ $\min _{x \in \bar{\Omega}} p(x) \leq p^{+}=\max _{x \in \bar{\Omega}} p(x)<+\infty$.

The variable exponent Lebesgue space $L^{p(\cdot)}(\Omega)$ is defined by

$$
\left\{u: \Omega \longrightarrow \mathbb{R}: \int_{\Omega}|u(x)|^{p(x)} d x<\infty\right\} .
$$

$L^{p(\cdot)}(\Omega)$ is endowed by the Luxemburg norm

$$
\left.|u|_{p(\cdot)}=\inf \left\{\mu>0: \int_{\Omega}\left|\frac{u(x)}{\mu}\right|^{p(x)} d x\right\} \leq 1\right\} .
$$

The generalized Lebesgue-Sobolev space $W^{1, p(\cdot)}(\Omega)$ is defined by

$$
W^{1, p(\cdot)}(\Omega)=\left\{u \in L^{p(\cdot)}(\Omega):|\nabla u| \in L^{p(\cdot)}(\Omega)\right\} .
$$

The space $W_{0}^{1, p(\cdot)}(\Omega)$ denotes the closure in $W^{1, p(\cdot)}(\Omega)$ of the set of all $W^{1, p(\cdot)}(\Omega)$-functions with compact support.

Proposition 2.1. (cf. [11])

(1) The spaces $L^{p(\cdot)}(\Omega), W_{0}^{1, p(\cdot)}(\Omega)$ are separable, reflexive, Banach spaces.

(2) The function space $\left(L^{p(\cdot)}(\Omega),\|u\|_{p(\cdot)}\right)$ is a separable and reflexive $B a$ nach space, where

$$
\left.\|u\|_{p(\cdot)}=\inf \left\{\mu>0: \int_{\Omega}\left|\frac{u(x)}{\mu}\right|^{p(x)} d x\right\} \leq 1\right\} .
$$

(3) Let $p_{1}^{\prime}$ be the function obtained by conjugating the exponent $p_{1}$ pointwise, that is $\frac{1}{p_{1}(x)}+\frac{1}{p_{1}^{\prime}(x)}=1$ for all $x \in \bar{\Omega}$, then $p_{1}^{\prime}$ belongs to $C_{+}(\bar{\Omega})$. For any $u \in L^{p_{1}(\cdot)}(\Omega)$ and $v \in L^{p_{1}^{\prime}(\cdot)}(\Omega)$, the following Hölder type inequality valid,

$$
\int_{\Omega}|u(x) v(x)| d x \leq\left(\frac{1}{p_{1}^{-}}+\frac{1}{p_{1}^{\prime-}}\right)\|u\|_{p_{1}(\cdot)}\|v\|_{p_{1}^{\prime}(\cdot)},
$$


where $L^{p_{1}^{\prime}(\cdot)}(\Omega)$ is the conjugate space of $L^{p_{1}(\cdot)}(\Omega)$.

(4) Let $p^{*}$ denote the critical variable exponent related to $p$, on $\bar{\Omega}$ and $k \geq 1$,

$$
\begin{gathered}
p^{*}(x)= \begin{cases}\frac{N p(x)}{N-p(x)} & p(x)<N, \\
+\infty & p(x) \geq N,\end{cases} \\
p_{k}^{*}(x)= \begin{cases}\frac{N p(x)}{N-k p(x)} & k p(x)<N, \\
+\infty & k p(x) \geq N .\end{cases}
\end{gathered}
$$

(5) For $p, q \in C_{+}(\bar{\Omega})$ in which $q(x) \leq p^{*}(x)$ for each $x \in \bar{\Omega}$, there is a continuous embedding

$$
W^{1, p(\cdot)}(\Omega) \hookrightarrow L^{q(\cdot)}(\Omega),
$$

the embedding is compact if $q(x)<p^{*}(x)$ for each $x \in \bar{\Omega}$.

(6) For every $u \in W_{0}^{1, p(\cdot)}(\Omega)$ the Poincaré inequality holds, i.e., there exists a positive constant $C_{p}$ in which

$$
\|u\|_{L^{p(\cdot)}(\Omega)} \leq C_{p}\|\nabla u\|_{L^{p(\cdot)}(\Omega)} .
$$

(7) If $p_{1}, p_{2} \in C(\bar{\Omega})$ and $1 \leq p_{1} \leq p_{2}$ in $\Omega$, then the embedding $L^{p_{2}(\cdot)} \hookrightarrow L^{p_{1}(\cdot)}$ is continuous.

(8) Since $p_{2}(x)<p_{1}(x)$ for any $x \in \Omega$ it follows that $W_{0}^{1, p_{1}(\cdot)}(\Omega)$ is continuously embedded in $W_{0}^{1, p_{2}(\cdot)}(\Omega)$.

(9) $\left(W_{0}^{1, p(\cdot)}(\Omega),\|\cdot\|\right)$ is a separable and reflexive Banach space. Define

$$
\|u\|=\inf \left\{\lambda>0: \int_{\Omega}\left[\left|\frac{\nabla u}{\lambda}\right|^{p(x)}+\left|\frac{u}{\lambda}\right|^{p(x)}\right] d x \leq 1\right\},
$$

for all $u \in W_{0}^{1, p(\cdot)}(\Omega)$.

Proposition 2.2. (cf. [11]) Let $p \in C_{+}(\bar{\Omega})$. Set $\varphi_{p(\cdot)}(u)=\int_{\Omega}|u(x)|^{p(x)} d x$. If $u,\left\{u_{n}\right\}_{n}$ are in $L^{p(\cdot)}(\Omega)$, when $1 \leq p_{-} \leq p_{+} \leq \infty$, then the following relations hold:

$(i)\|u\|_{p(\cdot)} \geq 1 \Rightarrow\|u\|_{p(\cdot)}^{p_{-}} \leq \varphi_{p(\cdot)} \leq\|u\|_{p(\cdot)}^{p_{+}}$,

$($ ii $)\|u\|_{p(\cdot)} \leq 1 \Rightarrow\|u\|_{p(\cdot)}^{p_{+}} \leq \varphi_{p(\cdot)} \leq\|u\|_{p(\cdot)}^{p_{-}}$. 
Proposition 2.3. (cf. [11]) Define $\Phi(u)=\int_{\Omega}\left[|\nabla u|^{p(x)}+|u(x)|^{p(x)}\right] d x$. For $u,\left\{u_{n}\right\}_{n} \in X$,

(i) $\|u\|<(=;>) 1 \Leftrightarrow \Phi(u)<(=;>) 1$,

(ii) $\|u\| \leq 1 \Rightarrow\|u\|^{p^{+}} \leq \Phi(u) \leq\|u\|^{p^{-}}$,

(iii) $\|u\| \geq 1 \Rightarrow\|u\|^{p^{-}} \leq \Phi(u) \leq\|u\|^{p^{+}}$,

(iv) $\left\|u_{n}\right\| \rightarrow 0 \Leftrightarrow \Phi\left(u_{n}\right) \rightarrow 0$,

(v) $\left\|u_{n}\right\| \rightarrow \infty \Leftrightarrow \Phi\left(u_{n}\right) \rightarrow \infty$.

Let $\eta: \partial \Omega \rightarrow \mathbb{R}$ be a measurable. Define the weighted variable exponent Lebesgue space by

$$
\begin{gathered}
L_{\eta(x)}^{p(x)}(\partial \Omega)=\{u: \partial \Omega \rightarrow \mathbb{R} \text { is measurable and } \\
\left.\int_{\partial \Omega}|\eta(x)||u|^{p(x)} d \sigma<\infty\right\},
\end{gathered}
$$

with the norm

$$
|u|_{(p(x), a(x))}=\inf \left\{\tau>0 ; \int_{\partial \Omega}|\eta(x)|\left|\frac{u}{\tau}\right|^{p(x)} d \sigma \leq 1\right\},
$$

where $d \sigma$ is the measure on the boundary.

Lemma 2.4. (cf. [8]) Let $\rho(x)=\int_{\partial \Omega}|a(x) \| u|^{p(x)} d \sigma$ for $u \in L_{a(x)}^{p(x)}(\partial \Omega)$ we have

$$
\begin{aligned}
& |u|_{(p(x), \eta(x))} \geq 1 \Rightarrow|u|_{(p(x), \eta(x))}^{p^{-}} \leq \rho(u) \leq|u|_{(p(x), \eta(x))}^{p^{+}}, \\
& |u|_{(p(x), \eta(x))} \leq 1 \Rightarrow|u|_{(p(x), \eta(x))}^{p^{+}} \leq \rho(u) \leq|u|_{(p(x), \eta(x))}^{p^{-}} .
\end{aligned}
$$

Define

$$
\begin{gathered}
p^{\partial}(x)=(p(x))^{\partial}:= \begin{cases}\frac{(N-1) p(x)}{N-p(x)} & p(x)<N, \\
+\infty & p(x) \geq N,\end{cases} \\
p^{\partial}(x)_{r(x)}:=\frac{r(x)-1}{r(x)} p^{\partial}(x),
\end{gathered}
$$

where $x \in \partial \Omega, r \in C(\partial \Omega, \mathbb{R})$ and $r(x)>1$. 
Proposition 2.5. (cf. [19], [20]) If $q \in C_{+}(\bar{\Omega})$ and $q(x)<p_{\partial}^{*}(x)$ for any $x \in \bar{\Omega}$, then the embedding from $W^{1, p(\cdot)}(\Omega)$ to $L^{q(x)}(\partial \Omega)$ is compact and continuous.

In this part we give a brief overview on some prerequisites on non-smooth analysis which are needed in the sequel. Let $X$ be a Banach space and $X^{\star}$ its topological dual. By $\|\cdot\|$ we will denote the norm in $X$ and by $\langle\cdot, \cdot\rangle_{X}$ the duality brackets for the pair $\left(X, X^{\star}\right)$. A function $f: X \rightarrow \mathbb{R}$ is said to be locally Lipschitz, if for every $x \in X$ there exists a neighbourhood $U$ of $x$ and a constant $K>0$ depending on $U$ such that $|f(y)-f(z)| \leq K\|y-z\|$ for all $y, z \in U$.

Let $f: X \rightarrow \mathbb{R}$ be a locally Lipschitz functional and $u, v \in X, v \neq 0$. We call the generalized directional derivative of $f$ in $u$ with respect to the direction $v$ the number

$$
f^{0}(u ; v)=\limsup _{w \rightarrow u, \lambda \rightarrow 0^{+}} \frac{f(w+\lambda v)-f(w)}{\lambda} .
$$

The generalized gradient of $f$ at $u \in X$ is defined by

$$
\partial f(u)=\left\{x^{\star} \in X^{\star}:\left\langle x^{\star}, v>_{X} \leq f^{0}(u ; v), \forall v \in X\right\},\right.
$$

which is a nonempty, convex and $w^{\star}$-compact subset of $X^{\star}$, where $<\cdot, \cdot>_{X}$ is the duality pairing between $X^{\star}$ and $X$.

A point $x \in X$ is said to be a critical point of the locally Lipschitz functional $f: X \rightarrow \mathbb{R}$ if $0 \in \partial f(x)$, that is, $f^{0}(x, v) \geq 0$, for every $v \in X \backslash\{0\}$.

Proposition 2.6. (cf. [6]) Let $h, g: X \rightarrow \mathbb{R}$ be locally Lipschitz functionals. Then, for every $u, v \in X$ the following conditions hold:

(1) $h^{0}(u ; \cdot)$ is subadditive, positively homogeneous;

(2) $\partial h$ is convex and weakly* compact;

(3) $(-h)^{0}(u ; v)=h^{0}(u ;-v)$;

(4) the set-valued mapping $h: X \rightarrow 2^{X^{*}}$ is weakly* u.s.c.;

(5) $h^{0}(u ; v)=\max _{u^{*} \in \partial h(u)}<u^{*}, v>$;

(6) $\partial(\lambda h)(u)=\lambda \partial h(u)$ for every $\lambda \in \mathbb{R}$;

(7) $(h+g)^{0}(u ; v) \leq h^{0}(u ; v)+g^{0}(u ; v)$;

(8) the function $m(u)=\min _{\nu \in \partial h(u)} \nu_{X^{*}}$ exists, and is lower semi continuous;

i.e., $\liminf u_{u \rightarrow u_{0}} m(u) \geq m\left(u_{0}\right)$.

To indicate the existence for solution of (1.1), we consider a functional $\mathcal{J}(u)=\phi(u)+\lambda I(u)-\mathcal{F}(u)$ associated to (1.1) which is defined by $\mathcal{J}(u): X \rightarrow \mathbb{R}$ such that $\forall u \in X$

$$
\phi(u)=\int_{\Omega} \frac{1}{p(x)}\left[|\nabla u|^{p(x)}+|u|^{p(x)}\right] d x, \quad I(u)=\int_{\Omega} \frac{1}{q(x)}|u|^{q(x)} d x,
$$




$$
\mathcal{F}(u)=\int_{\partial \Omega} F(x, u(x)) d \sigma
$$

Proposition 2.7. (cf. [22]) The operator $\phi^{\prime}(u): X \rightarrow X^{\star}$ is given by

$$
<\phi^{\prime}(u), v>=\int_{\Omega}\left(|\nabla u|^{p(x)-2} \nabla u \nabla v+|u|^{p(x)-2} u v\right) d x, \quad \forall u, v \in X .
$$

and satisfies the following properties:

(i) $\phi^{\prime}$ is continuous, bounded and strictly monotone.

(ii) $\phi^{\prime}$ is of $\left(S_{+}\right)$type.

(iii) $\phi^{\prime}$ is a homeomorphism.

Definition 2.8. The functional $\mathcal{J}: X \rightarrow X^{\star}$ verifies the $\left(\mathcal{S}_{+}\right)$property if for any weakly convergence sequence $\left\{u_{n}\right\}_{n} \subset X$ to $u$ in $X$ and

$$
\limsup _{n \rightarrow \infty}<\mathcal{J}\left(u_{n}\right), u_{n}-u>\leq 0
$$

implies that $\left\{u_{n}\right\}_{n}$ converges strongly to $u$ in $X$.

We say that $\mathcal{J}$ satisfies the nonsmooth Palais-Smale condition, if any sequence $\left\{x_{n}\right\}_{n \geq 1} \subseteq X$ such that $\left\{\mathcal{J}\left(x_{n}\right)\right\}_{n \geq 1}$ is bounded and $m\left(x_{n}\right)=\min \left\{\left\|x^{*}\right\|_{*}\right.$ : $\left.x^{*} \in \partial \mathcal{J}\left(x_{n}\right)\right\} \Rightarrow 0$ as $n \rightarrow \infty$, has a strongly convergent subsequence.

\section{Main Result}

We assume that $F: \Omega \times \mathbb{R} \rightarrow \mathbb{R}$ is a Carathéodory function, which is locally Lipschitz in the second variable and satisfying the following properties:

$\left(F_{1}\right)$ For almost all $x \in \Omega$ and all $v \in \partial F(x, t)$, we have $|v| \leq \alpha(x)$ with $\alpha(x) \in L_{+}^{\infty}(\Omega)=\left\{f \in L^{\infty}(\Omega): \operatorname{ess}_{\inf _{x \in \Omega}} f(x)>0\right\}$;

$\left(F_{2}\right) \quad|F(x, s)| \leq \epsilon|s|^{q^{+}}+c(\epsilon)$ for all $(x, s) \in \Omega \times \mathbb{R}, q^{-} \leq q^{+}<\left(p^{*}\right)^{-}$;

$\left(F_{3}\right) \lim _{|u| \rightarrow 0^{+}} \frac{f(x, u)}{u^{p^{-}-1}}=\infty$ uniformly for $x \in \partial \Omega$.

We state our main result in the following theorem.

Theorem 3.1. Suppose that conditions $F_{1}-F_{3}$ are satisfied. Then there exists $\lambda_{*}$ such that for any $\lambda \in\left(0, \lambda_{*}\right)$, problem 1.1 has a sequence of nontrivial solutions $\left\{u_{n}\right\}$ and $u_{n} \rightarrow 0$ as $n \rightarrow \infty$.

For proving our main result, we need some lemmas and a proposition. 
Lemma 3.2. Suppose that $F_{i}, i=1,2,3$ hold and $\lambda \in\left(-\infty, \frac{q^{-} \lambda_{*}}{p^{+}}\right)$. Then functional I satisfies the Palais-Smale condition.

Proof. Let $\left\{u_{n}\right\}_{n \geq 1} \subseteq W_{0}^{1, p(\cdot)}(\Omega)$ be a sequence such that $\left\{\mathcal{J}\left(u_{n}\right)\right\}_{n \geq 1}$ is bounded and $m\left(u_{n}\right) \rightarrow 0$ as $n \rightarrow \infty$. We will show that the sequence $\left\{u_{n}\right\}_{n \geq 1} \subseteq$ $W_{0}^{1, p(\cdot)}(\Omega)$ is bounded.

Assume by contradiction by passing to a subsequence, we can suppose that $\left\|u_{n}\right\| \rightarrow \infty$ as $n \rightarrow \infty$.

Let $z_{n}=\frac{u_{n}}{\left\|u_{n}\right\|}$ for all $n \geq 1$. Up to a subsequence, we assume that

$$
\begin{aligned}
& z_{n} \rightarrow z \text { in } L^{p(\cdot)}(\Omega), \\
& z_{n}(x) \rightarrow z(x) \text { a.e. in } \Omega, \\
& z_{n} \rightarrow z \text { in } X,
\end{aligned}
$$

as $n \rightarrow \infty$.

Applying Lebourg's mean value theorem (cf. [6]), imply the existence of

$v_{n}(x) \in \partial\left(x, \theta_{x} u_{n}(x)\right)$ with $0<\theta_{x}<1$ for almost all $x \in \partial \Omega$ and for all $n \geq 1$,

such that

$$
\left|F\left(x, u_{n}(x)\right)-F(x, 0)\right|=\theta_{x} u_{n}(x) \leq\left|\theta_{x}\right| \cdot\left|u_{n}(x)\right| .
$$

From hypothesis $F_{1}$,

$$
\left|F\left(x, u_{n}(x)\right)\right| \leq|F(x, 0)|+\alpha(x)\left|u_{n}(x)\right| \leq \alpha_{1}+\alpha_{1}\left|u_{n}(x)\right|,
$$

for some $\alpha_{1}, \alpha_{2}>0$.

Then

$\left|\int_{\partial \Omega} \frac{F\left(x, u_{n}(x)\right)}{\left\|u_{n}\right\|^{\varsigma}}\right| \leq \int_{\partial \Omega} \frac{\left|F\left(x, u_{n}(x)\right)\right|}{\left\|u_{n}\right\|^{\varsigma}} \leq \int_{\partial \Omega} \frac{\alpha_{1}+\alpha_{1}\left|u_{n}(x)\right|}{\left\|u_{n}\right\|^{\varsigma}} \leq \frac{\alpha_{3}}{\left\|u_{n}\right\|^{\varsigma}}+\frac{\alpha_{4}}{\left\|u_{n}\right\|^{\varsigma-1}}$,

$\forall \varsigma>1$ and for some $\alpha_{3}, \alpha_{4}$.

Hence,

$$
\frac{F\left(x, u_{n}(x)\right)}{\left\|u_{n}\right\|^{\varsigma}} \rightarrow 0 \quad \text { as } \quad n \rightarrow \infty .
$$

Due to $\left|\mathcal{J}\left(u_{n}\right)\right| \leq M$ and $\left\|u_{n}\right\| \rightarrow \infty$ for all $n \geq 1$, we can assume that $\left\|u_{n}\right\| \geq 1$ and

$$
\int_{\Omega} \frac{1}{p(x)}\left|\nabla u_{n}\right|^{p(x)} d x-\int_{\Omega} \frac{\lambda}{q(x)}\left|u_{n}\right|^{q(x)}-\int_{\partial \Omega} F\left(x, u_{n}(x)\right) d x \leq M .
$$


These are two cases.

Case 1. $\lambda>0$.

$$
\int_{\Omega} \frac{1}{p^{+}}\left|\nabla u_{n}\right|^{p(x)} d x-\int_{\Omega} \frac{\lambda}{q^{-}}\left|u_{n}\right|^{q(x)}-\int_{\partial \Omega} F\left(x, u_{n}(x)\right) d x \leq M .
$$

By the definition of $\lambda_{*}$, and the continuous embedding of $X$ into $L^{q(\cdot)}(\Omega)$

$$
\int_{\Omega}\left(\frac{1}{p^{+}}-\frac{\lambda}{q^{-} \lambda_{*}}\right)\left|\nabla u_{n}\right|^{p(x)} d x-\int_{\partial \Omega} F\left(x, u_{n}(x)\right) d x \leq M .
$$

We consider now two subcases.

Subcase 1.1. Suppose that there exists a sequence $\left\{u_{n}\right\}_{n \geq 1} \subseteq L^{p(\cdot)}(\Omega)$ such that

$$
\left\|\nabla u_{n}\right\|_{p(\cdot)} \leq 1 \text { for all } n \geq 1
$$

According to proposition 2.3 it follows that

$$
\int_{\Omega}\left(\frac{1}{p^{+}}-\frac{\lambda}{q^{-} \lambda_{*}}\right)\left|\nabla u_{n}\right|_{p(\cdot)}^{p^{+}} d x-\int_{\partial \Omega} F\left(x, u_{n}(x)\right) d x \leq M .
$$

Dividing the inequality by $\left\|u_{n}\right\|^{p^{+}}$

$$
\int_{\Omega}\left(\frac{1}{p^{+}}-\frac{\lambda}{q^{-} \lambda_{*}}\right)\left|\nabla z_{n}\right|_{p(\cdot)}^{p^{+}} d x-\int_{\partial \Omega} \frac{F\left(x, u_{n}(x)\right)}{\left\|u_{n}\right\|^{p^{+}}} d x \leq \frac{M}{\left\|u_{n}\right\|^{p^{+}}} .
$$

By the fact that $\frac{1}{p^{+}}-\frac{\lambda}{q^{-} \lambda_{*}}>0$ and (3.1), it leads to

$$
\nabla z_{n} \rightarrow 0 \quad \text { in } \quad L^{p(\cdot)}(\Omega)
$$

Subcase 1.2. If subcase 1.1 does not hold, then we can suppose that there exists a subsequence $\left\{u_{n}\right\}_{n \geq 1} \subseteq L^{p(\cdot)}(\Omega)$ such that

$$
\left\|\nabla u_{n}\right\|_{p(\cdot)}>1 \text { for all } n \geq 1 \text {. }
$$

According to the proposition 2.3, it follows that

$$
\int_{\Omega}\left(\frac{1}{p^{+}}-\frac{\lambda}{q^{-} \lambda_{*}}\right)\left|\nabla u_{n}\right|_{p(x)}^{p^{-}} d x-\int_{\partial \Omega} F\left(x, u_{n}(x)\right) d x \leq M .
$$

Dividing the inequality by $\left\|u_{n}\right\|^{p^{-}}$

$$
\int_{\Omega}\left(\frac{1}{p^{+}}-\frac{\lambda}{q^{-} \lambda_{*}}\right)\left|\nabla z_{n}\right|_{p(\cdot)}^{p^{-}} d x-\int_{\partial \Omega} \frac{F\left(x, u_{n}(x)\right)}{\left\|u_{n}\right\|^{p^{-}}} d x \leq \frac{M}{\left\|u_{n}\right\|^{p^{-}}} .
$$


If we pass to the limit as $n \rightarrow \infty$ in (3.7) and relation (3.1),

$$
\nabla z_{n} \rightarrow 0 \quad \text { in } \quad L^{p(\cdot)}(\Omega)
$$

Consequently, by (3.6) and (3.8)

$$
\nabla z_{n} \rightarrow 0 \quad \text { in } \quad L^{p(\cdot)}(\Omega) .
$$

Case 2. Let $\lambda \leq 0$. Using (3.2),

$$
\int_{\Omega} \frac{1}{p^{+}}\left|\nabla u_{n}\right|^{p(x)} d x-\int_{\partial \Omega} F\left(x, u_{n}(x)\right) d x \leq M .
$$

Again, as already shown, there are two subcases.

Subcase 2.1. Suppose that there exists a $\left\{u_{n}\right\}_{n \geq 1} \subseteq L^{p(\cdot)}(\Omega)$ such that

$$
\left\|\nabla u_{n}\right\|_{p(\cdot)} \leq 1 \text { for all } n \geq 1 \text {. }
$$

According to proposition 2.3

$$
\frac{1}{p^{+}}\left\|\nabla u_{n}\right\|_{p(\cdot)}^{p^{+}}-\int_{\partial \Omega} F\left(x, u_{n}(x)\right) d x \leq M .
$$

Dividing the inequality by $\left\|u_{n}\right\|^{p^{+}}$

$$
\int_{\Omega} \frac{1}{p^{+}}\left|\nabla z_{n}\right|_{p(\cdot)}^{p^{+}} d x-\int_{\partial \Omega} \frac{F\left(x, u_{n}(x)\right)}{\left\|u_{n}\right\|^{p^{+}}} d x \leq \frac{M}{\left\|u_{n}\right\|^{p^{+}}} .
$$

By the fact that $\frac{1}{p^{+}}>0$ and $(3.1)$,

$$
\nabla z_{n} \rightarrow 0 \quad \text { in } \quad L^{p(\cdot)}(\Omega)
$$

Subcase 2.2. If subcase 1.1 does not hold, then we can suppose that there exists a subsequence $\left\{u_{n}\right\}_{n \geq 1} \subseteq L^{p(\cdot)}(\Omega)$ such that

$$
\left\|\nabla u_{n}\right\|_{p(\cdot)}>1 \text { for all } n \geq 1 \text {. }
$$

In view of proposition 2.3 it follows that

$$
\int_{\Omega}\left(\frac{1}{p^{+}}-\frac{\lambda}{q^{-} \lambda_{*}}\right)\left|\nabla u_{n}\right|_{p(\cdot)}^{p^{-}} d x-\int_{\partial \Omega} F\left(x, u_{n}(x)\right) d x \leq M .
$$

With similar arguments as in the proof of relation in subcase 2.1, we can show that

$$
\nabla z_{n} \rightarrow 0 \quad \text { in } \quad L^{p(\cdot)}(\Omega)
$$


These two cases imply that

$$
\nabla z_{n} \rightarrow 0 \quad \text { in } \quad L^{p(\cdot)}(\Omega)
$$

Using $\lambda_{*}$ in (3.3) in another way. Similarly, dividing argument in two cases

$$
z_{n} \rightarrow 0 \quad \text { in } L^{p(\cdot)}(\Omega)
$$

From (3.9), (3.13) and (3.14),

$$
z_{n} \rightarrow 0 \text { in } X
$$

Denote by $z_{n}=\frac{u_{n}}{\left\|u_{n}\right\|}$ then $\left\|z_{n}\right\|=1$ for all $n \geq 1$ which represents a contradiction with (3.15). So $\left\{u_{n}\right\}_{n \geq 1} \subseteq X$ is bounded. Hence, by passing to a subsequence if necessary, we may assume that

$$
\begin{aligned}
& u_{n} \rightarrow u \quad \text { weakly in } X, \\
& u_{n} \rightarrow u \quad \text { in } \quad L^{q(\cdot)}(\Omega), \\
& u_{n} \rightarrow u \text { in } L^{r(\cdot)}(\Omega) .
\end{aligned}
$$

Since $\partial \mathcal{J}\left(u_{n}\right) \subseteq X^{\star}$ is weakly compact and the norm functional in a Banach space is weakly lower semicontinuous, by the Weierstrass theorem, we can assume that $u_{n}^{*} \in \partial \mathcal{J}\left(u_{n}\right)$ such that

$$
\left\|u_{n}^{*}\right\|_{*}=m\left(u_{n}\right), \quad \text { for } \quad n \geq 1 .
$$

Then, for every $n \geq 1$,

$$
u_{n}^{*}=\phi^{\prime} u_{n}-\lambda\left|u_{n}\right|^{q(x)-2} u_{n}-v_{n}^{*}
$$

where $v_{n}^{*} \in \partial \mathcal{F}\left(u_{n}\right) \subseteq L^{r^{\prime}(\cdot)}(\Omega)$ for $n \geq 1$. Choosing the sequence $\left\{u_{n}\right\}_{n \geq 1} \subseteq$ $W_{0}^{1, p(\cdot)}(\Omega)$,

$$
\left|\left\langle u_{n}^{*}, v\right\rangle\right| \leq \epsilon_{n}\|v\| \quad \text { for all } \quad v \in W_{0}^{1, p(\cdot)}(\Omega)
$$

with $\epsilon_{n} \searrow 0$.

Putting $\nu=u_{n}-u$ in (3.18) and using (3.17),

$$
\left\langle\phi^{\prime} u_{n}, u_{n}-u\right\rangle-\lambda \int_{\Omega}\left|u_{n}\right|^{q(x)-2} u_{n}\left(u_{n}-u\right)(x) d x-\int_{\partial \Omega} v_{n}^{*}(x)\left(u_{n}-u\right)(x) d x
$$




$$
\leq \epsilon_{n}\left\|u_{n}-u\right\|
$$

Since $\left\{u_{n}\right\}_{n \geq 1} \subseteq L^{p(\cdot)}(\Omega)$ is bounded, using proposition 2.1 and the compactly embedded $W_{0}^{1, p(\cdot)}(\Omega)$ into $L^{q(\cdot)}(\Omega)$ then $\left\{u_{n}\right\}$ converges strongly to $u$ in $L^{q(\cdot)}(\Omega)$ we deduce that

$\lambda \int_{\Omega}\left|u_{n}(x)\right|^{q(x)-2} u_{n}(x)\left(u_{n}-u\right)(x) d x \leq \lambda\left(\frac{1}{q^{-}}+\frac{1}{q^{\prime-}}\right)\left\|\left|u_{n}\right|^{q(x)-1}\right\|_{q^{\prime}(\cdot)}\left\|u_{n}-u\right\|_{q(\cdot)} \rightarrow 0$

as $n \rightarrow \infty$, where $\frac{1}{q(x)}+\frac{1}{q^{\prime}(x)}=1$. The following relation

$$
\int_{\partial \Omega} v_{n}^{*}(x)\left(u_{n}-u\right)(x) d x \rightarrow 0 \quad \text { as } \quad n \rightarrow \infty
$$

holds true.

If we pass to the limit in (3.19) as $n \rightarrow \infty$

$$
\limsup _{n \rightarrow \infty}\left\langle\phi^{\prime} u_{n}, u_{n}-u\right\rangle \leq 0 .
$$

Taking into account that the operator $\phi$ has the $\left(S_{+}\right)$property,

$$
u_{n} \rightarrow u \text { in } X
$$

This proves that $f$ satisfies the Palais-Smale condition.

Definition 3.3. Let $X$ be a Banach space and denote

$\Gamma:=\{A \subset X \backslash\{0\}: A$ is closed in $X$ and symmetric with respect to the orgin $\}$.

For $A \in \Gamma$, we define genus $\gamma(A)$ as follows:

$$
\gamma(A)=: \inf \left\{m \in \mathbb{N}: \exists \varphi \in C\left(A, \mathbb{R}^{m} \backslash\{0\}\right),-\varphi(x)=\varphi(-x)\right\} .
$$

If there is no mapping $\varphi$ as above for any $m \in \mathbb{N}$, then $\gamma(A)=+\infty$. Let $\gamma_{k}$ denote the family of closed symmetric subsets $A$ of $X$ such that $0 \notin A$ and $\gamma(A) \geq k$.

For the convenience of the readers, we summarize the properties of a genus. We refer the readers to (cf. [3]) for the proof of the next proposition.

Proposition 3.4. Let $A$ and $B$ be closed symmetric subsets of $X$ which do not contain the origin. Then the following hold.

(1) If there exists an odd continuous mapping from $A$ to $B$, then $\gamma(A) \leq \gamma(B)$; 
(2) If there exists an odd homeomorphism from $A$ to $B$, then $\gamma(A)=\gamma(B)$;

(3) If $\gamma(B)<\infty$, then $\gamma(\overline{A \backslash B}) \geq \gamma(A)-\gamma(B)$;

(4) If $A$ is compact, then $\gamma(A)<\infty$ and $\gamma\left(N_{\delta}(A)\right)=\gamma(A)$ for $\delta>0$ small enough;

(5) The $n$-dimensional sphere $S^{n}$ has a genus of $n+1$ by the Borsuk-Ulam theorem.

The following version of the symmetric mountain-pass lemma is due to Kajikiya (cf. [14]).

Lemma 3.5. Let $X$ be an infinite-dimensional space and $\mathcal{J} \in C^{1}(X, \mathbb{R})$ satisfy (B1) and (B2) as follows;

(B1) $\mathcal{J}(u)$ is even, bounded from below, $\mathcal{J}(0)=0$ and $\mathcal{J}(u)$ satisfies the PalaisSmale condition.

(B2) For each $k \in \mathbb{N}$, there exists an $A_{k} \in \Gamma_{k}$ such that $\sup _{u \in A_{k}} \mathcal{J}(u)<0$.

Then either $\left(R_{1}\right)$ or $\left(R_{2}\right)$ as the following holds.

$\left(R_{1}\right)$ There exists a sequence $\left\{u_{k}\right\}$ such that $\mathcal{J}^{\prime}\left(u_{k}\right)=0, \mathcal{J}\left(u_{k}\right)<0$ and $\left\{u_{k}\right\}$ converges to zero.

$\left(R_{2}\right)$ There exist two sequences $\left\{u_{k}\right\}$ and $\left\{v_{k}\right\}$ such that $\mathcal{J}^{\prime}\left(u_{k}\right)=0, \mathcal{J}\left(u_{k}\right)<$ $0, u_{k} \neq 0, \lim _{k \rightarrow \infty} u_{k}=0, \mathcal{J}^{\prime}\left(v_{k}\right)=0, \mathcal{J}\left(v_{k}\right)<0, \lim _{k \rightarrow \infty} v_{k}=0$ and $\left\{v_{k}\right\}$ converges to a non-zero limit.

Remark 3.6. From lemma 3.5, there is a sequence $\left\{u_{k}\right\}$ of critical points such that $\mathrm{J}\left(u_{k}\right) \leq 0, u_{k} \neq 0$ and $\lim _{k \rightarrow \infty} u_{k}=0$.

Let $\mathcal{J}(u)$ be the functional defined as before, we have

$$
\begin{aligned}
\mathcal{J}(u) & =\int_{\Omega} \frac{\left(|\nabla u|^{p(x)}+a(x)|u(x)|^{p(x)}\right) d x}{p(x)}-\lambda \int_{\Omega} \frac{|u|^{q(x)}}{q(x)} d x-\int_{\partial \Omega} F(x, u(x)) d \sigma \\
& \geq \frac{1}{p^{+}}\|u\|_{p(\cdot)}^{p^{+}}-\frac{\lambda}{q^{-}}\|u\|_{q(\cdot)}^{q^{+}}-\int_{\partial \Omega}\left(\epsilon|u|^{q^{+}}+c(\epsilon)|\partial \Omega|\right) d \sigma \\
& \left.\geq \frac{1}{p^{+}}\|u\|_{p(\cdot)}^{p^{+}}-\left(\frac{\lambda}{q^{-}}+\epsilon\right)\|u\|_{q(\cdot)}^{q^{+}}-c(\epsilon)|\partial \Omega|\right) \\
& =A\|u\|^{p^{+}}-B\|u\|_{q(\cdot)}^{q^{+}}-C,
\end{aligned}
$$

where $\epsilon=\frac{\lambda}{q^{-}}, A=\frac{1}{p^{+}}, B=\frac{2 \lambda}{q^{-}}, C=c \frac{\lambda}{q^{-}}|\partial \Omega|$, for any $u \in W_{0}^{1, p(\cdot)}(\Omega)$ with $\|u\|_{p(\cdot)}<1$. We define

$$
p(t)=A t^{p^{+}}-B t^{q^{+}}-C
$$


then $\mathcal{J}(u) \geq p\left(\|u\|_{p(\cdot)}\right)$. By the definition of $p(t)$ and the fact that $p^{+}<q^{+}$, $p(t)$ attains its positive maximum, there exists

$$
R_{1}=\left(\frac{p^{+} A}{q^{+} B}\right)^{\frac{1}{p^{+}-q^{+}}}
$$

such that

$$
d_{1}=p\left(R_{1}\right)=\max _{t \geq 0} p(t)>0 .
$$

Hence, for $d_{0} \in\left(0, d_{1}\right)$, we may find $R_{0}<R_{1}$ such that $p\left(R_{0}\right)=d_{0}$. For these values $R_{1}$ and $R_{0}$, we can choose a smooth function $h(t)$ defined as follows

$$
h(t)=\left\{\begin{array}{lc}
1 & 0 \leq t \leq R_{0} \\
\frac{A t^{p^{+}}-C-d_{1}}{B t^{q^{+}}} & t \geq R_{1} \\
C^{\infty}, h(t) \in[0,1], & R_{0} \leq t \leq R_{1} .
\end{array}\right.
$$

Let $\rho(u)=h\left(\|u\|_{p(\cdot)}\right)$ and consider the perturbation of $\mathcal{J}(u)$.

$$
\begin{aligned}
& \tilde{\mathcal{J}}(u)=\int_{\Omega} \frac{\left(|\nabla u|^{p(x)}+a(x)|u(x)|^{p(x)}\right) d x}{p(\cdot)} \\
& -\rho(u) \int_{\Omega} \frac{\lambda|u|^{q(x)}}{q(x)} d x-\rho(u) \int_{\partial \Omega} F(x, u(x)) d \sigma . \\
& \tilde{\mathcal{J}}(u) \geq A\|u\|_{p(\cdot)}^{p^{+}}-B \rho(u)\|u\|_{p(\cdot)}^{q^{+}}-C=\bar{p}\left(\|u\|_{p(\cdot)}\right),
\end{aligned}
$$

where $\bar{p}(t)=A t^{p^{+}}-B h(t) t^{q^{+}}-C$ and

$$
\bar{p}(t)=\left\{\begin{array}{lc}
p(t) & 0 \leq t \leq R_{0} \\
d_{1} & t \geq R_{1} .
\end{array}\right.
$$

Proposition 3.7. Let $\overline{\mathcal{J}}(u)$ be in (3.22). Then

(1) $\overline{\mathcal{J}} \in C^{1}(X, \mathbb{R})$ and $\overline{\mathcal{J}}$ is even and bounded from below,

(2) If $\overline{\mathcal{J}}(u)<d_{0}$ then $\bar{p}(\|u\|)<d_{0}$, consequently, $\|u\|_{p(\cdot)}<R_{0}$ and $\mathcal{J}(u)=$ $\overline{\mathcal{J}}(u)$,

(3) Suppose that $F_{1}-F_{3}$ hold, then $\overline{\mathcal{J}}(u)$ satisfies the $(P S)$ condition.

Lemma 3.8. Assume that $\left(F_{3}\right)$ holds. Then for any $k \in \mathbb{N}$, there exists $\delta=\delta(k)>0$ such that $\gamma\left(\left\{u \in W_{0}^{1, p(\cdot)}(\Omega) ; \tilde{\mathcal{\partial}}(u) \leq-\delta(k)\right\} \backslash\{0\}\right) \geq k$. 
Proof. $\left(F_{3}\right)$ implies that

$$
F(x, \iota u) \geq N(\iota)(\iota u)^{p^{-}}, N(\iota) \rightarrow \infty \text { as } \iota \rightarrow 0 .
$$

Let $E_{k}$ be a $k$-dimensional subspace of $W_{0}^{1, p(\cdot)}(\Omega)$. Hence, for any $u \in E_{k}$ with $\|u\|_{p(\cdot)}=1$ and $\iota$ small enough, for $0<\iota<\min \left\{R_{0}, 1\right\}$

$$
\begin{gathered}
\overline{\mathcal{J}}(\iota u)=\mathcal{J}(\iota u) \\
=\int_{\Omega} \iota^{p(x)} \frac{\left(|\nabla u|^{p(x)}+a(x)|u(x)|^{p(x)}\right) d x}{p(x)}-\int_{\Omega} \iota^{p(x)} \frac{\lambda|u|^{q(x)}}{q(x)} d x-\int_{\partial \Omega} F(x, \iota u(x)) d \sigma \\
\leq \int_{\Omega} \frac{1}{p^{-}} \iota^{p^{-}} \frac{\left(|\nabla u|^{p(x)}+a(x)|u(x)|^{p(x)}\right) d x}{p(x)}-\frac{1}{q^{+}} \iota^{q^{+}} \int_{\Omega} \frac{\lambda|u|^{q(x)}}{q(x)} d x-N(\iota) \iota^{p^{-}} \int_{\partial \Omega}|u|^{p^{-}} d \sigma .
\end{gathered}
$$

Since $E_{k}$ is a space of finite dimension, all the norms in $E_{k}$ are equivalent. We define

$$
\begin{aligned}
& A_{k}=\inf \left\{\int_{\Omega}|u|^{q(x)} d x: u \in E_{k},\|u\|_{p(\cdot)}=1\right\}>0, \\
& B_{k}=\inf \left\{\int_{\Omega}|u|^{p^{-}} d x: u \in E_{k},\|u\|_{p(\cdot)}=1\right\}>0 .
\end{aligned}
$$

In view of (3.24), it implies that

$$
\begin{gathered}
\bar{\jmath}(u) \leq \frac{1}{p^{-}} \iota^{p^{-}}-\frac{1}{q^{+}} \iota^{q^{+}} A_{k}-N(\iota) \iota^{p^{-}} B_{k} \\
\leq \iota^{p^{-}}\left(\frac{1}{p^{-}}-N(\iota) B_{k}\right)-\frac{1}{q^{+}} \iota^{q^{+}} A_{k}=-\delta(k)<0, \text { as } \iota \rightarrow 0,
\end{gathered}
$$

since $\lim _{|\iota| \rightarrow 0} N(\iota)=+\infty$. It results that

$$
\left\{u \in E_{k} ;\|u\|_{p(\cdot)}=\iota\right\} \subset\left\{u \in W_{0}^{1, p(\cdot)}(\Omega) ; \bar{\partial}(u) \leq-\delta(k)\right\} \backslash\{0\} .
$$

Proof of Theorem 3.1: we define

$$
c_{k}=\inf _{A \in \Gamma_{k}} \sup _{u \in A} \overline{\mathcal{J}}(u) .
$$

According to proposition 3.7(1) and lemma 3.8, it results that $-\infty<c_{k}<0$. Therefore, assumptions (B1) and (B2) of lemma 3.5 are verified. As a result, there exists a sequence $\left\{u_{k}\right\}$ converging to zero. Hence, theorem 3.1 follows by proposition $3.7(2)$. 
INFINITELY MANY SOLUTIONS FOR A CLASS OF HEMIVARIATIONAL

\section{References}

[1] M. Alimohammady, F. Fattahi, Existence of solutions to hemivaritional inequalities involving the $p(x)$-biharmonic operator, Electron. J. Diff. Equ., 2015(2015), no. 79, 1-12.

[2] M. Allaoui, Existence of solutions for a Robin problem involving the $p(x)$-Laplacian, Applied Mathematics E-Notes, 14(2014), 107-115.

[3] A. Ambrosetti, A. Malchiodi, Nonlinear Analysis and Semilinear Elliptic Problems, Cambridge Studies in Advanced Mathematics 104. Cambridge: Cambridge University Press, 2007.

[4] G. Bonannoa, D. Motreanu, P. Winkert, Variational-hemivariational inequalities with small perturbations of nonhomogeneous Neumann boundary conditions, J. Math. Anal. Appl. 381(2011), 627-637.

[5] G. Bonannoa, P. Winkert, Multiplicity results to a class of variationalhemivariational inequalities, Topological methods in nonlinear analysis, 43(2)(2014), 493-516.

[6] F. H. Clarke, Optimization and nonsmooth analysis, John Wiley \& Sons, New York, 1983.

[7] G. D'AGUÌ, Second-order boundary-value problems with variable exponents, Electronic Journal of Differential Equations, 2014(2014), no. 68, $1-10$.

[8] S. G. Dend, Eigenvalues of the p(x)-laplacian Steklov problem, J. Math. Anal. Appl., 339 (2008), 925-937.

[9] L. Diening, Riesz potential and Sobolev embeddings on generalized Lebesgue and Sobolev spaces $L^{p(x)}(\Omega)$ and $W^{1, p(x)}(\Omega)$, Math. Nachr. 268(2004), 31-43.

[10] D. E. Edmunds, J. Rákosník, Density of smooth functions in $W^{k, p(x)}(\Omega)$, Proc. R. Soc. A, 437(1992), 229-236.

[11] X. L. Fan, D. Zhao, On the generalized Orlicz - Sobolev space $W^{m, p(x)}(\Omega)$, J. Gansu Educ. College, 12(1998), 1-6.

[12] S. Heidarkhani, G. Afrouzi, A. Hadjian, J. henderson, Existence of infinitely many anti-periodic solutions for second-order impulsive differential inclusions, Electronic Journal of Differential Equations, 2013(2013), no. $97,1-13$. 
[13] A. Kristály, Infinitely many radial non-radial solutions for a class of hemivariational inequalities, Rocky Mountain Journal of Mathematics, Vol. 35, 4(2005), 1173-1190.

[14] R. Kajikiya, A critical-point theorem related to the symmetric mountain-pass lemma and its applications to elliptic equations, J. Funct. Analysis, 225(2005), 352-370.

[15] L. Li, C. Tang, Existence and multiplicity of solutions for a class of $p(x)$-biharmonic equations, Acta Mathematica Scientia, 33B(1)(2013), 155-170.

[16] G. Sun, K. Teng, Existence and multiplicity of solutions for a class of fractional Kirchhoff-type problem, Math. Commun. 183 19(2014), 183194.

[17] M. C. Wei, C. L. Tang, Existence and Multiplicity of Solutions for $p(x)$-Kirchhoff-Type Problem in $\mathbb{R}^{N}$, Bull. Malays. Math. Sci. Soc. (2) 36(3)(2013), 767-781.

[18] Y. Ye, C.L. Tang, Multiplicity of solutions for elliptic boundary value problems, Electronic Journal of Differential Equations, 2014(2014), No. $140,1-13$.

[19] X. L. Fan, Regularity of minimizers of variational integrals with $p(x)$-growth conditions, Ann. Math. Sinica, 17A(5)(1996), 557-564.

[20] O. Kováčik , J. Rákosnínk, On spaces $L^{p(x)}$ and $W^{1, p(x)}$, Czechoslovak Math. J., 41(1991), 592-618.

[21] X. L. Fan, Q. H. Zhang, Existence of solutions for $p(x)$-Laplacian Dirichlet problems , Nonlinear Anal., 52(2003), 1843-1852.

[22] L. Li, L. Ding, W. W. Pan, Existence of multiple solutions for a $p(x)$-biharmonic equation, Electron J Differ Equ, 2013(2013), No. 139, $1-10$.

[23] D. Motreanu, P. Winkert, Variational-hemivariational inequalities with nonhomogeneous neumann boundary condition, Le Matematiche, Vol. (2010) Fasc. II, 109-119 doi: 10.4418/2010.65.2.12

[24] V. Rădulescu, Nonlinear elliptic equations with variable exponent: old and new, Nonlinear Analysis, Theory, Methods and Applications, 121(2015), 336-369. 
INFINITELY MANY SOLUTIONS FOR A CLASS OF HEMIVARIATIONAL

[25] V. Rădulescu, D. Repovs, Partial differential equations with variable exponents, Variational Methods and Qualitative Analysis, CRC Press, Taylor \& Francis Group, Boca Raton, 2015.

Fariba Fattahi,

Department of Mathematics,

University of Mazandaran, Babolsar, IRAN.

Email: F.Fattahi@stu.umz.ac.ir

Mohsen Alimohammady,

Department of Mathematics,

University of Mazandaran, Babolsar, IRAN.

Email:Amohsen@umz.ac.ir 